\title{
La especialización agrícola en la historia rural europea: recursos, mercados y espacios (siglos XVIII-XX)
}

Agricultural specialization in European rural history: resources, markets and spaces $\left(18^{\text {th }}-20^{\text {th }}\right.$ centuries $)$

\author{
Laurent Herment \\ CNRS, Centre de recherches historiques, EHESS, Francia \\ lherment@ehess.fr \\ Niccolò Mignemi \\ CNRS, Laboratoire interdisciplinaire des énergies de \\ demain, Université de Paris, Francia \\ niccolo.mignemi@u-paris.fr
}

\begin{abstract}
Resumen:
En el presente artículo se cuestiona la noción de especialización agrícola a partir de los debates sobre las innovaciones técnicas y el desarrollo socioeconómico rural entre los siglos XVIII y XX. Para ello, los autores se basan en los resultados de las investigaciones que han actualizado la historia rural europea durante las últimas décadas y proponen tres posibles lecturas para entender cómo los cultivos y las actividades especializadas han transformado la organización de las explotaciones y de los territorios. En primer lugar, parten de la síntesis pionera de la historiadora Joan Thirsk sobre la agricultura alternativa para analizar las distintas maneras de describir y rechazar este fenómeno desde el punto de vista de la producción. La segunda lectura se centra en el desarrollo de las redes comerciales, las cadenas de suministro y los sectores industriales concebidos, en cierta medida, para modelar la huella espacial de los sistemas especializados. En tercer lugar, y desde el punto de vista de estos sistemas, estudian las dinámicas contemporáneas de intensificación productiva, racionalización técnica y selección socioeconómica.
\end{abstract}

PALABRAS ClAVE: Especialización agrícola, Organización espacial, Innovación, Sectores agroalimentarios, Europa occidental.

\section{ABstract:}

The article analyzes the notion of agricultural specialization looking at the debates on technical innovations and socio-economic development in the countryside between the $18^{\text {th }}$ and the $20^{\text {th }}$ century. It is based on the research literature that has renewed European rural history during the past decades. Three different perspectives are proposed to explore how specialized crops and activities transformed farming and regional organization. Firstly, the pioneering historical work on alternative agricultures by Joan Thirsk serves to compare various definitions and approaches to specialization from the production side. Secondly, the article questions how changes in commercial networks, supply chain and industrial activities can shape the spatial footprint of the specialized rural systems. Thirdly, these systems become the fieldwork for observing the contemporary dynamics of agricultural intensification, technical rationalization, and socio-economic selection.

KEYWORDS: Agricultural specialization, Spatial organization, Innovation, Agrifood chain, Western Europe.

La noción de especialización agrícola puede parecer una obviedad en la medida en que es posible oponerla a la de diversificación. En otras palabras, una explotación se considera especializada si se centra en una determinada producción o actividad en lugar de dar cabida a diferentes opciones. Desde este punto de vista, el término constituye un sinónimo de monocultivo o actividad única. Sin embargo, una explotación puede acoger dos producciones principales, como por ejemplo la vid y el trigo, la ganadería lechera y la cerealicultura o la producción forrajera. A veces, el segundo tipo de producción es solo un complemento del primero. El juicio sobre el carácter especializado de dicha explotación variará en función del peso que se otorgue a cada actividad, o de si solo se tienen en cuenta los productos alimenticios comercializados. 
El presente artículo pretende plantear estas dinámicas y mecanismos de funcionamiento. Ello nos permitirá explorar la complejidad y profundidad de la noción de especialización agrícola a lo largo de los últimos tres siglos. De este modo nos referiremos a una serie de modelos y marcos imperativos surgidos en diferentes momentos de la reflexión económica. Nuestro análisis se centrará principalmente en los trabajos de la historiografía europea que, durante los últimos veinte años (Lains y Pinilla, 2009; Béaur, 2012; Herment, 2019), han estudiado las transformaciones de los mundos rurales y agrícolas del Viejo Continente en relación con los fenómenos más generales del desarrollo económico y de las innovaciones científico-técnicas.

La oposición entre las ventajas de la producción a gran escala y las virtudes de la pequeña explotación ha ocupado durante mucho tiempo los debates contemporáneos sobre las vías de crecimiento y las transformaciones sociotécnicas en el campo. La teoría de la especialización agrícola permite contemplar estas evoluciones de diferentes maneras. En primer lugar, la especialización nos invita a estudiar el papel de las crisis, los diferentes efectos que estas pueden provocar y la variedad de posibles respuestas. Por otro lado, permite abordar las relaciones contradictorias, pero necesarias, entre los agricultores, los mercados locales ylas redes comerciales de larga distancia. En tercer lugar, nos permite replantear el lugar que ocupa la agricultura en el proceso de industrialización, más allá de ser la principal fuente de materias primas destinadas a la transformación de productos alimenticios, textiles, etc. ${ }^{1}$

Para analizar hasta qué punto los cultivos especializados han sido un terreno de innovación agronómica y social en los campos de la Europa occidental desde el siglo XVIII, nuestro artículo se dividirá en tres partes. En primer lugar, redefiniremos los diferentes paradigmas del fenómeno desde el punto de vista de la producción y ello a partir de la síntesis pionera de la historiadora Joan Thirsk. En segundo lugar, estudiaremos cómo el desarrollo de los sistemas de suministro y los circuitos comerciales han definido la huella espacial de los sistemas especializados. Por último, analizaremos las dinámicas de intensificación productiva, racionalización técnica y selección socioeconómica -centrales en las últimas transformaciones agrarias europeas- desde el punto de vista de la especialización.

\section{Cultivo alternativo, Producción especializada, aCtividad CentraL}

La obra Alternative agriculture, de Joan Thirsk (1997), no tuvo la acogida que merecía fuera del Reino Unido. Un ejemplo de ello es, en Francia, las críticas de Jean-Pierre Poussou (1999) y Gérard Béaur (2009), a las que cabe añadir el reciente volumen de la colección Rural History in Europe (RURHE) de la editorial Brepols (Béaur, 2020). Las reflexiones de Jean-Pierre Poussou (1999 y 2020) permiten comprender el escaso interés de los estudios rurales franceses por abordar el concepto de "agriculturas alternativas" avanzado por JoanThirsk. Según la historiadora británica, durante los períodos de descenso de los precios de las producciones dominantes (cereales y ganadería, fundamentalmente de carne), los campesinos buscan otras opciones. Se podría decir que Alternative agriculture constituye la continuación de los trabajos de Bernard H. Slicher van Bath y sus epígonos sobre los factores demográficos, económicos y tecnológicos del desarrollo, ${ }^{2}$ según los cuales los sistemas agrarios se adaptan con mayor o menor rapidez, completa o parcialmente, a la evolución de las relaciones de precios de las diferentes producciones. La originalidad de Joan Thirsk radica en el hecho de que opone una agricultura dominante (centrada en la producción de cereales y proteínas animales) a la aparición de cultivos alternativos que pueden conducir a una dinámica de especialización más o menos sostenible. En definitiva, el principal interés de esta perspectiva reside en poder cuestionar un modelo único de innovación y de crecimiento agrícola, a partir de la constatación de la íntima relación de las estructuras sociales y las actividades productivas en el campo (Thirsk, 1999, p. 8; Dyer, 2000; Hoyle, 2004; Jones y Dyer, 2016).

La modesta acogida de las tesis de Joan Thirsk, tanto en Francia como en otros países, puede explicarse de dos modos. En primer lugar, la vid y el olivo, que ocupan tanto espacio e implican a gran parte de la población en el centro, y, sobre todo, en el sur de Europa, no figuran en la tesis de la autora. Para los franceses, españoles, 
portugueses, italianos, griegos, parte de los alemanes, suizos, austriacos, húngaros, etc., hablar en términos de oposición entre la cerealicultura y la ganadería, por una parte, y la agricultura alternativa, por otra, no tiene especial sentido. La vid y el vino son algo más, mucho más a veces, que una alternativa. El olivo dibuja el rastro visible de un paisaje mediterráneo ocupado y trabajado por las familias campesinas. En segundolugar, al destacar la importancia de las crisis como impulsoras de cultivos alternativos, Thirsk corre el riesgo de ocultar el papel crucial de las innovaciones agronómicas, los mercados y el dinamismo del campesinado en todo el mundo. Esta crítica nos invita a superar o a resituar la noción de alternativas (Béaur, 2009 y 2020). Es cierto que los sistemas agrarios europeos se caracterizan desde muy pronto -ya en el siglo XVIII- por fenómenos de especialización cuya geografía es raramente fortuita y a menudo persistente. Podemos encontrar muchos ejemplos en el espacio francés y en otros espacios nacionales o regionales de la Edad Moderna. ${ }^{3}$

Así pues, si consideramos las vertientes industriales y comerciales de la especialización, la oposición binaria entre mainstream agriculture . alternative agriculture introducida por Joan Thirsk pierde relevancia. De hecho, la especialización está presente tanto al apostar por un alternative crop como al favorecer la mainstream agriculture en forma de ganadería -lechera o de carne- o de cerealicultura. A menudo, la producción de trigo, centeno, cebada o avena, además de ser una opción alimentaria, constituye en sí misma un abanico de opciones de especialización con fines comerciales. Así pues, las alternative agricultures constituyen solo una de las posibles vías de especialización.

Para definir los límites de esta observación es necesario retomar la estructura de los sistemas agrícolas y de pastoreo. Durante la Edad Moderna -y también en la Edad Media y en la Antigüedad- la principal preocupación de las explotaciones es el autoconsumo. Se trata, incluso, de un elemento crucial en el desarrollo de los procesos productivos. La paja, el forraje, la madera, la ceniza, el estiércol, etc., se producen y consumen en las mismas explotaciones. Por otro lado, como indica Maurice Aymard (1983), entre los mercados y el campesinado se establece "una relación contradictoria de inclusión (a menudo forzada) y de exclusión (de sus beneficios)" ${ }^{4}$. Alimentada por los circuitos del autoconsumo y demandada por los mercados, la especialización se inserta en la cotidianidad de las explotaciones, hasta el punto de impedir la oposición binaria entre especialización y autoconsumo.

En este contexto, la especialización es un complemento de los ingresos esenciales para la subsistencia de las familias, accesible a los agricultores a través de los mercados. Así pues, la preocupación por garantizar las necesidades de la explotación no puede ignorar la posibilidad de invertir en una producción particular para su comercialización. Esto se debe a dos razones. En primer lugar, desde un punto de vista agrícola, económico y social, la especialización tiene un fuerte impacto en las tierras, especialmente en el ciclo de rotación de cultivos, en los modos y ritmos de movilización de la mano de obra, y en la elección y organización de las plantaciones. En segundo lugar, la especialización es inherente al establecimiento de redes comerciales, que drenan una parte de los recursos locales y atraen otros desde el exterior (Béaur, 2009; Herment y Antoine, 2016). Estos dos aspectos - el de la producción y el de la relación con el mercado- constituyen, en cierta manera, los dos niveles del despegue de la "especialización" agrícola hasta finales del siglo XIX.

Frente a la mera intuición, que no permite entender las evoluciones y los resultados del fenómeno, resulta indispensable superar la idea de que la especialización es necesariamente sinónimo de monocultivo o actividad única. En ocasiones, la agricultura de plantación puede ajustarse a este modelo, aunque con el riesgo de agotar los recursos necesarios para garantizar su sostenibilidad (Ouerfelli, 2008; Tomich, dos Santos Gomes y Gomes da Cunha, 201 1; Uekötter, 2014; Barral, 2015). Sin embargo, si nos centramos en la Edad Moderna, las contribuciones recogidas en Antoine (2016) demuestran que el fenómeno de especialización puede, y en la mayoría de los casos debe, formar parte de la rotación de cultivos y de la asociación de actividades. Para la inmensa mayoría de los agricultores, productores, enólogos, ganaderos, horticultores y arboricultores, se trata, por lo tanto, de fomentar complementariedades -en términos de recursos, tareas y ritmos estacionales- que solo pueden garantizarse en las explotaciones o territorios gracias a los complejos sistemas de policultivo o policultivo ganadero. En la introducción de la obra citada más arriba, Laurent 
Herment y Annie Antoine proponen observar este fenómeno desde la perspectiva del cultivo o actividad central (pivot, en francés) para así distinguir los mecanismos que permiten que una especialización se dé en un lugar y se mantenga a lo largo del tiempo (Herment y Antoine, 2016, pp. 26-28).

En este sentido, "central" puede entenderse de dos maneras: como el cultivo o actividad más importante en términos de empleo y recursos invertidos, o como la parte de la producción que se comercializa. Si tomamos el primer ejemplo, nos arriesgamos a dejar de lado lo que durante mucho tiempo ha sido la peculiaridad de la especialización en el marco de los sistemas de policultivo. En muchos casos, los cereales o la ganadería dominan, o incluso reemplazan, a otras producciones, ya que son esenciales para el autoconsumo y la continuidad de los procesos productivos. Si la visión de Joan Thirsk presupone la incompatibilidad entre cultivo dominante y alternativo, el enfoque propuesto por Laurent Herment y Annie Antoine ofrece nuevas perspectivas, que pueden discutirse de diferentes maneras según el contexto. Por un lado, el cultivo o actividad central podría considerarse como el punto de partida de la rotación. No obstante, esta perspectiva supone rotaciones y cultivos anuales, y, normalmente, las inversiones se extienden durante mucho más tiempo en numerosos sistemas centrados en la ganadería, la viticultura o la arboricultura frutal. Por otro, el término pivot puede ayudarnos a identificar el cultivo o la actividad sobre la que se organizan el espacio rural, las relaciones sociales y las redes comerciales. En dichas configuraciones, esta noción a menudo revela el sistema multifuncional y pluriactivo que ha acompañado el desarrollo de muchos sistemas agrarios en un contexto protoindustrial durante siglos (Hubscher, 1979-1980; Dewerpe, 1985; Rinaudo, 1987; Garrier y Hubscher, 1988; Villani, 1989; Bevilacqua, 1990; Mayaud, 1999; Lorenzetti, 2010).

Si entendemos la actividad central de la segunda manera, y, por lo tanto, nos centramos en la finalidad comercial de la misma, podemos observar procesos originales y formas de especialización diversas. Destacan la cerealicultura -especialmente de cebada- en algunas regiones de Anglia Oriental (Dickinson, 1934), de avena en la cuenca parisina (Moriceau y Postel-Vinay, 1992), de trigo en Beauce (Farcy, 1989), la transformación del lino en el interior de Flandes (Ronsijn, 2019), o incluso la ganadería en Bretaña, la cría de ganado lechero en la Baja Normandía o en el País de Bray, y el engorde de animales en Limosín (Delhoume, 2009) o en Mayenne (Antoine, 1995). También destacan la rubia, planta tintórea anterior al auge del cultivo de hortalizas, frutas y verduras en el Condado Venesino (Mesliand, 1989), la fruticultura cerca de grandes centros urbanos (Quellier, 2003), la sericicultura en toda la península italiana (Cafagna, 1989; Federico, 1994), el cáñamo en la Emilia Romaña y Venecia (Martini, 2000; Celetti, 2007), y los olivares y vergeles de la cuenca mediterránea (Aymard, 1978; Bevilacqua, 1989; Morilla Critz, 1995; Infante-Amate, 2014). Así pues, aun teniendo en cuenta las prioridades de producción, la perspectiva del cultivo o actividad central no es víctima de un determinismo geológico o pedológico. Identifica climas, estructuras y tendencias, pero, sobre todo, se pregunta por las potencias económicas, las innovaciones tecnológicas y el entramado territorial que han diseñado las diferentes formas de especialización agrícola durante siglos.

Esto nos invita a cambiar de perspectiva. Ya no podemos analizar la especialización como una alternativa puramente productiva, que decidimos o no llevar a cabo. Debemos reflexionar sobre su aplicación a partir de la doble relación que establece con su vinculación territorial. Por un lado, como actividad, la especialización se inscribe en territorios determinados, en los que desempeña un papel unificador y, al mismo tiempo, ayuda a definir las fronteras. Por otro lado, si la consideramos como una fuente de productos destinados al mercado o a la transformación industrial, su proyección hacia el exterior se debe a las redes de suministro y a las infraestructuras comerciales. Para entender el carácter y la evolución de los cultivos especializados, es necesario abrir y examinar en toda su amplitud la caja negra de los sistemas agrarios locales. ${ }^{5}$

\section{Producción Y SUMinistro: Distancia Y LOCALIZACióN}

La consideración del espacio, o, mejor dicho, de los diferentes planos en los que se inscriben las especializaciones agrícolas, debemos en gran medida al economista y terrateniente alemán Johan 
Heinrich von Thünen, interesado por la localización de los factores de producción y por la circulación de los recursos entre áreas complementarias. Asimismo, von Thünen hace hincapié en las interconexiones e intercambios para demostrar que la capacidad que tiene la tierra para producir riqueza no es solo algo intrínseco y natural. Su obra permite cuestionar y ampliar las reflexiones de Thomas Malthus (1815) y de David Ricardo (1817), a quien debemos la ley de los rendimientos decrecientes. ${ }^{6}$ Según Ricardo, el crecimiento demográfico va acompañado del cultivo de nuevas tierras cada vez menos fértiles, lo que provoca un descenso de la productividad - marginal y media- y un crecimiento de la renta del suelo. El mundo rural ricardiano se sitúa en un terreno puramente agronómico, donde el único criterio discriminatorio es el diferente grado de fertilidad del suelo. ${ }^{7}$

No estamos exagerando si afirmamos que el espacio que concibe Ricardo no entiende de coordenadas geográficas: la heterogeneidad de los espacios y de los cultivos son solo elementos secundarios con el consiguiente riesgo de desaprovechar las oportunidades de expansión productiva (Grantham, 1999). Por el contrario, la localización de las tierras es un elemento central en el modelo del Estado aislado propuesto por von Thünen (1826). La variable fundamental ya no es la calidad del suelo, sino la distancia entre las tierras y el centro de la ciudad. Johan Heinrich von Thünen parte de la hipótesis de una llanura completamente fértil y sin límites, pero aislada, en donde, sin embargo, los cultivos se distribuyen en función de la demanda del mercado central, las dificultades de transporte (volumen, peso, naturaleza perecedera, etc.) y los costes del transporte de productos. Aunque el modelo es estático, el autor afirma la sucesión de círculos concéntricos de especialización agrícola. En primer lugar, la horticultura y la producción lechera ocupan el círculo más cercano a la ciudad, así como las zonas boscosas. El siguiente círculo es el de la cerealicultura, hasta llegar a las tierras más alejadas, en donde se encuentra la ganadería extensiva.

Según Ricardo, la posición de una tierra puede justificarse desde un punto de vista económico si tenemos en cuenta las desigualdades presentes entre los suelos. En cambio, para von Thünen, las relaciones espaciales son el principal elemento de diferenciación en un universo homogéneo, uniforme y tridimensional. Aunque es cierto que la pérdida de fertilidad del suelo es la base de la teoría de la renta diferencial de Ricardo, esta no tiene en cuenta la variedad de posibles cultivos. La teoría de la renta de localización de von Thünen supone un cambio de perspectiva, según el cual el valor de la mercancía depende de la distancia y de las condiciones de transporte entre los lugares donde esta se produce y se consume. En los debates sobre la idea de que la renta es la expresión de las condiciones naturales e intrínsecas de la tierra, el economista alemán proporciona los medios analíticos necesarios para concebir el proceso de especialización en un marco sistémico. Al margen de las elecciones individuales en una explotación, es necesario comprender la organización de las orientaciones productivas en un territorio o región. Sin embargo, las redes comerciales todavía ocupan un lugar secundario en este esquema, ya que siguen siendo el complemento de la geografía.

En su conocida obra Nature's Metropolis (1991), William Cronon retoma el modelo de von Thünen, cuestiona la noción de frontera en la historia de los Estados Unidos y, sobre todo, identifica las interacciones que se dan en el contínuum rural-urbano. El nacimiento de Chicago como plataforma agroalimentaria a nivel mundial se estudia desde el punto de vista de las influencias recíprocas entre el reacondicionamiento de los paisajes rurales norteamericanos, transformados por la explotación de los territorios al Oeste, y la apertura de nuevas rutas comerciales. La evolución de las relaciones mercantiles y el crecimiento de la rentabilidad de las inversiones conducen a dinámicas de especialización que consiguen que los ecosistemas locales de gestión de recursos tiendan a integrarse en una red de cuencas regionales y en circuitos de suministro internacionales. Las ventajas naturales ya no bastan para explicar la geografía de las orientaciones productivas, que William Cronon sitúa en una jerarquía de concentraciones demográficas y redes comerciales.

En esta síntesis, dedicada al caso norteamericano, la distancia se alza como un elemento esencial, no solo en el funcionamiento, sino también en la evolución de los sistemas agroalimentarios. Tiene como objetivo definir el espacio a partir del desarrollo de infraestructuras técnicas y económicas, y el cambio en el tiempo gracias a las innovaciones en los transportes y las comunicaciones. Si, años después, el modelo de Heckscher- 
Ohlin celebra los beneficios del libre comercio y de la especialización a escala internacional, la globalización de los mercados agrícolas contribuye a la creación de ciclos desde el siglo XIX; unos ciclos, cada vez más largos, que integran las opciones de cada explotación y en los que se suceden las etapas de producción, procesamiento, y comercialización. Frente a estas dinámicas, los aranceles aduaneros protegen en gran medida la mainstream agriculture de cereales. En cambio, estas políticas no se aplican a la mayoría de los cultivos especializados de gran valor añadido, en los que la presión de la competencia mundial consigue acelerar la innovación científicotécnica y la mejora de la organización de los mercados y sectores agroindustriales (Morilla Critz, Olmstead y Rhode, 1999 y 2000; Pinilla y Ayuda, 2009).

La influencia de las áreas y circuitos de suministro en la evolución de la productividad y la especialización agrícola ha sido, y es, de especial interés para los historiadores desde hace mucho tiempo. Más concretamente, se ha estudiado la capacidad de reacción de los espacios rurales ante las demandas de los centros urbanos, así como sus interacciones con el desarrollo de las actividades no agrícolas de la Europa preindustrial (Grantham, 1989 y 1997). Estos enfoques restan importancia a las distancias y a los medios de transporte, presentes en el modelo de von Thünen, y se centran en las economías externas determinadas por la localización de las actividades productivas, hasta el punto de que algunos autores buscan en ellas el origen de la "pequeña divergencia" entre regiones europeas (Kopsidis y Wolf, 2012). La demanda de los mercados se convierte así en el principal motor de los procesos de intensificación y especialización. A la demanda urbana y protoindustrial le siguen las nuevas oportunidades de los mercados a escala nacional e internacional (Wrigley, 2006; Pinilla, 2009).

Sin embargo, a finales del siglo XIX, los poderes públicos adquieren un papel fundamental en este ámbito: empiezan apoyando las innovaciones tecnológicas, biológicas e institucionales para poco a poco intervenir de manera más directa en las políticas de modernización del sector agroalimentario (Moser y Varley, 2014). Estos reflexionan sobre cómo integrar las orientaciones productivas locales en dinámicas de desarrollo más generales. A principios del siglo XX, por ejemplo, el padre de la geografía francesa, Paul Vidal de La Blache (1910) se pronunció sobre un proyecto de reforma descentralizadora con la posibilidad de promover un mosaico de "agrupaciones regionales" que definiera el perfil económico del conjunto del territorio francés. Este debate le permite reflexionar sobre las diferentes maneras de concebir la división espacial y sobre las consecuencias particulares de los criterios adoptados para ello. Si la noción de región natural identifica tradicionalmente conjuntos homogéneos resultantes del entorno físico e histórico, Vidal de La Blache ya insistía en la necesidad de enriquecer esta representación, que no considera adecuada para captar todos los desafíos con que nos retan los cambios que se dan en la movilidad de los factores y en los sistemas de transporte. En este punto se trataría de concebir nuevos marcos -como la región económica- que nos permitieran integrar espacios diversos en relación con su tejido productivo, aunque geográficamente cercanos y sobre todo interdependientes, como consecuencia de las redes de intercambio y de las circulaciones biofísicas.

Más allá de las acciones individuales, estos programas pretenden reforzar las economías externas en los territorios. Mientras la nueva economía geográfica prestaba especial atención a este último aspecto (Krugman, 1991), Alfred Marshall ya había demostrado en Principles of Economics (1890), que la concentración de industrias especializadas en un espacio presenta una serie de ventajas. En primer lugar, un mercado de trabajo extenso y cualificado, gracias a la circulación de las habilidades locales; por otro lado, intercambios de bienes impulsados por la proximidad espacial de los productores; finalmente, medios de transmisión de la información y de intercambio de experiencias. A partir del término "distrito industrial", el economista italiano Giacomo Becattini retoma los trabajos de Marshall con el objetivo de poner en relación la permanencia en un lugar de las actividades productivas con un medio sociocultural e institucional. Este enfoque, elaborado a partir de ejemplos manufactureros, ha desencadenado una discusión sobre su posible aplicación en el terreno agrícola y rural (Becattini, 2000 y 2001; De Benedictis, 2000; Musotti, 2000; Iacoponi, 2001). Se han propuesto diferentes soluciones con el objetivo de identificar estos sistemas 
productivos locales (Iacoponi, 1990; Cecchi, 1992) y en Italia se ha instaurado un marco legislativo. Los distritos se han convertido en una herramienta analítica y operativa para movilizar las sinergias locales al servicio de las políticas agrícolas y del desarrollo rural en los territorios en donde se espera que los productos y paisajes conformen una identidad común.

Más allá de estas de variantes prácticas, nuestro interés por los distritos se basa en la posibilidad de contemplar una posible organización del espacio en términos de ecosistema de actividades. Estas pistas parecen especialmente fructíferas para pensar en términos de articulación entre las especializaciones agrícolas, las actividades artesanales y la manufactura, que acompaña la expansión de los sectores agroalimentarios entre los siglos XIX y XX. Como indican los últimos trabajos, es necesario estudiar el arraigo local y las posibilidades de explotación como parte de los circuitos de suministro rediseñados a raíz de la difusión de los centros de almacenamiento, el desarrollo de las fábricas de transformación de productos y despojos, y la construcción de redes de transporte para el envío de productos alimenticios a distancias cada vez mayores (Drouard y Williot, 2007; Segers, Bieleman y Buyst, 2009). Las zonas de producción se distancian cada vez más de los lugares de consumo, y, además, han de competir con nuevas técnicas como la deshidratación, la refrigeración y la conservación (Morilla Critz, Olmstead y Rhode, 2000).

Paradójicamente, esta dinámica globalizadora de los circuitos comerciales va acompañada de una nueva preocupación: la consolidación de un producto en un contexto ecológico, económico y cultural delimitado en el espacio. Así, la marca se convierte en un instrumento capaz de sintetizar este vínculo y alterar el valor de un producto en el mercado, con un impacto que las políticas del sector agroalimentario no tardan en controlar mediante el recurso de las denominaciones. Este hecho explica el interés por deshacer el vínculo que se establece entre una especialización y el territorio en donde se implanta y evoluciona. Aunque a menudo se resalta la vocación natural para dar cuenta de dicha herencia, los investigadores han demostrado que no basta para explicarlo todo. En algunas regiones, el trabajo de los actores económicos, las preocupaciones de las comunidades locales o la visión de los planificadores contribuyen a consolidar o promover una estructura original entre recursos naturales, prácticas culturales y técnicas más o menos artesanales (Garrabou, Tello y Cussó, 2010; Chiapparino, 2015). La especialización agrícola, aquí, no es más que una parte de un proceso mucho más vasto del que se sirven el mecanismo de certificación y la noción de región en busca de patrimonialización y valorización (Delfosse, 2007 y 2011; Chiapparino y Tedeschi, 2017).

Este interesante cambio de perspectiva nos hace pensar, de manera colectiva, en las condiciones de partida y las posibilidades del ser humano para transformar un medio, más allá del modelo monocéntrico y estático que plantea von Thünen. Si bien la especialización no surge de manera natural, la promoción de sus cualidades "naturales" es solamente uno de los aspectos que permiten pensar en su proyección espacial. En realidad, los cultivos especializados no se limitan a una disposición específica generada por un conjunto de condiciones geomorfológicas y pedoclimáticas, de reservas de materia viva disponibles (vegetal y animal) y de elementos técnicos (medios de tracción y de trabajo, material para el tratamiento de las semillas, invernaderos, silos, etc.). Aunque es cierto que estos elementos son determinantes, lo que verdaderamente define los cultivos especializados es la coexistencia de estos medios en los lugares de producción y en los circuitos comerciales. El auge de la especialización de la mantequilla en Dinamarca a finales del siglo XIX muestra cómo la orientación ganadera se apoyó en las importaciones de cereales, en las nuevas oportunidades surgidas de la demanda inglesa y en la disposición de un sistema sociotécnico como garantía de calidad de la producción cooperativa (Henriksen y O'Rourke 2005; Henriksen, 2009). Así pues, el suministro de las industrias y mercados, a mayor o menor distancia, constituye un criterio esencial para explicar cómo las especializaciones agrícolas pueden modelar y seleccionar las explotaciones de un territorio. 


\section{Cuando la especialización SE CONVIERTe EN Monocultivo}

El lugar que ocupa la especialización en las dinámicas de desarrollo agrícola merece una reflexión desde el punto de vista de las profundas transformaciones de los últimos dos siglos. Paul Bairoch (1989) menciona tres revoluciones agrícolas en Europa y en Norteamérica en la época contemporánea. En primer lugar, distingue el aumento de la productividad gracias a las innovaciones técnicas y biológicas de Inglaterra desde el siglo XVIII. A continuación hace hincapié en la internacionalización de los mercados y los circuitos de suministro como fuerza motriz de las dinámicas que se dan en cada vez más países a partir de la década de 1850. Para terminar, nos muestra cómo la adopción masiva de insumos industriales y máquinas conduce a un alza de los rendimientos. Este es un crecimiento que, al destinar las inversiones a las tecnologías intensivas en capital, consigue compensar los efectos del éxodo rural y de la caída de los activos agrícolas en el siglo XX. Al mismo tiempo, los países del Sur ven cómo la Revolución verde consolida su posición hegemónica gracias a un modelo de desarrollo del sector, basado en tres pilares: la selección de semillas, el uso de fertilizantes y la irrigación (Cleaver, 1972).

Estas perspectivas subrayan los diferentes factores que han favorecido el crecimiento de la producción y el fuerte aumento de la productividad agrícola. Como consecuencia, la especialización ocupa a menudo un segundo plano respecto a las cuestiones mencionadas más arriba, pero no por ello deja de ser un argumento recurrente tanto en los debates de los actores de la época como en los análisis de los historiadores. Podríamos incluso afirmar que constituye un imperativo absoluto que se consolida progresivamente en la agenda modernizadora del sector agroalimentario. Por otro lado, Adam Smith sitúa la división y la especialización del trabajo como la base del crecimiento industrial, pero la aplicación de estos conceptos en agricultura no ha logrado el consenso durante un largo período. Las controversias entre los partidarios de la gran y pequeña explotación van acompañadas de las críticas de numerosos expertos, que no tardan en calificar la especialización agrícola de "doctrina" y "consigna”, arriesgándose a poner en peligro los equilibrios tradicionales de la rotación de cultivos y las asociaciones productivas. A partir de la década de 1860, las crisis económicas y sanitarias revelan los límites de este modelo, como señalan las observaciones de Édouard Zacharewicz - profesor de agricultura en el departamento francés de Vaucluse- en un territorio que se enfrenta al declive de la rubia, de la sericicultura y de la vid, así como a la reconversión en explotaciones frutícolas y hortícolas (Mesliand, 1989, pp. 195-196).

La diversificación ha sido durante mucho tiempo una opción casi inevitable para garantizar la fertilidad del suelo, la gestión de plagas y la protección frente a posibles enfermedades. Sin embargo, en la segunda mitad del siglo XX se observa un cambio de perspectiva, con la difusión de los fertilizantes y pesticidas que acaban alterando la organización interna y los equilibrios metabólicos de la agricultura. La internacionalización de las redes de suministro y la creciente importancia de los factores de producción de origen industrial dan la sensación de que ya es posible forzar los límites locales y emanciparse de los ciclos naturales de reproducción de recursos. Estas evoluciones se basan en la difusión de los fertilizantes, los tratamientos y la selección de semillas, pero provocan al mismo tiempo la ruptura de los mecanismos tradicionales de rotación y diversificación. Son unos mecanismos cada vez más olvidados en busca de una mayor eficacia y rentabilidad. Los avances científico-técnicos que mejoran la variedad, el acondicionamiento del suelo y la fumigación, así como las innovaciones funcionales en los sectores agroindustriales y en la organización de los mercados, refuerzan esta tendencia. Los imperativos de la racionalización e intensificación ocupan un lugar cada vez más importante en la definición de los equilibrios de las explotaciones. Finalmente, estos imperativos priman sobre la diversidad como mecanismo de control de imprevistos y sobre la protección de las interacciones ecosistémicas, reducidas a gastos suplementarios (Rosset y Altieri, 1997).

Paralelamente, la especialización de las explotaciones y de los espacios regionales se convierte en una prioridad para las políticas agrícolas, que prometen guiar las orientaciones productivas de acuerdo con los programas de desarrollo del sector (Dumont, 1952). Los Estados, las administraciones y las organizaciones 
profesionales actúan a diferentes niveles para acompañar la emergencia de verdaderas zonas productivas, capaces de inscribir las transformaciones locales en procesos evolutivos a gran escala (Durbiano, 2000). Las posibilidades parecen existir, al menos en la teoría. En función de las características de cada territorio, algunos expertos imaginan la posibilidad de impulsar especializaciones parciales, es decir un conjunto de cultivos bien situados, pero, sin embargo, susceptibles de padecer cambios, según las condiciones económicas (Bordas, 1954, p. 32). No obstante, esta visión parece algo compleja de gestionar en comparación con el modelo de la especialización de monocultivos, mucho más fácil de generalizar. La evolución es evidente. Así lo observamos en los diferentes trabajos, que hacen hincapié en el fin de la policultura y de las economías agropecuarias a favor de sistemas que contemplan un número limitado de opciones con el objetivo de garantizar un mejor rendimiento productivo y un punto de equilibrio entre las explotaciones (van der Ploeg et al., 2009). A partir de los medios disponibles y la productividad del trabajador, Marcel Mazoyer y Laurence Roudart proponen un marco analítico con el que seguir el curso de las especializaciones contemporáneas y de las relocalizaciones de las actividades a escala mundial (1997).

El incremento de las dinámicas de internacionalización e industrialización agrícolas desde mediados del siglo XIX (Kautsky, 1899) favorece la difusión de la especialización de cultivos en algunos territorios donde las condiciones pedoclimáticas y las redes comerciales son favorables. Aun así, el auge de este movimiento depende de las oportunidades ofrecidas por las inversiones - públicas y privadas - en las infraestructuras (transporte, gestión hidráulica, unidades de almacenamiento y transformación, etc.), así como de la promoción por parte de las administraciones, instituciones científicas y organismos profesionales (Bevilacqua, 1991; Maat, 2001; Pan-Montojo, 2005; Failla y Fumi, 2006; Valceschini, 2016). Las condiciones naturales garantizan ciertas ventajas comparativas y economías de escala que la extensión de los circuitos de suministro, las innovaciones científico-técnicas y la debilidad de las barreras comerciales sobre ciertos productos convierten en elementos de competencia internacional (Federico, 2005; Pinilla y Ayuda, 2009). Estas evoluciones redefinen las distancias, los ciclos estacionales, los flujos de materiales y las mercancías. Las posibilidades de acceso a las redes de irrigación, a las infraestructuras de transporte y a las fábricas de transformación pasan a ser variables estratégicas (Bethemont, 1972; Calatayud, 2004; Williot, 2010). Estas conducen a la exclusión de las producciones más aisladas y en peor posición, lo que se traduce en espirales de especialización cada vez más acentuadas y situadas en el espacio. Un ejemplo de ello es el proceso de regionalización progresiva de las producciones vitivinícolas y hortícolas (Durbiano, 1997).

Como bien indica Joan Thirsk, las oportunidades comerciales constituyen un pilar esencial para explicar el desarrollo de los cultivos especializados. Cultivos que pueden acoger tanto productos alimenticios destinados al consumo urbano, como materias primas industriales, productos de lujo para el mercado nacional o para su exportación. No obstante, a partir del período de entreguerras, con la llegada del modelo productivista, en agricultura se inicia una nueva etapa. Este modelo favorece la idea de la abundancia como elemento democratizador del acceso a los alimentos y mercancías, combinando la modernización técnica y la reglamentación económica. Podría considerarse un equivalente agrícola del fordismo en la industria, que vuelve a configurar las relaciones entre la ciencia, el Estado y las organizaciones profesionales con el objetivo de promover la producción y el consumo en masa (Allaire, 1996; Bonneuil, Denis y Mayaud, 2008; Moser y Varley, 2013; Brunier, 2018). Si los cultivos especializados están a la vanguardia de este movimiento es gracias a su temprana organización en sectores agroindustriales y en nodos (web). Así lo explican algunos autores en términos de interconexión entre los actores y las circulaciones que unen espacios y sectores diversos. De esta manera, los cultivos especializados constituyen un terreno de innovaciones tecnológicas y prácticas comerciales que permiten la difusión progresiva de estándares comunes y la fabricación de calidad como variables estratégicas en el funcionamiento de los mercados (Stanziani, 2005; Bernard de Raymond, 2013). En este sentido, el cultivo de cítricos, por ejemplo, ha llamado especialmente la atención de historiadores de diferentes países mediterráneos (Calatayud, 1989; Lupo, 1990; Karlinsky, 2005; Garrido, 2010). Cultivados tradicionalmente en la cuenca mediterránea, a lo largo del último siglo, los cítricos se han convertido en 
materias primas para las industrias y en productos accesibles a un gran público. Así pues, para responder al aumento progresivo de la demanda de estos cultivos, los vergeles han redibujado completamente el paisaje de ciertas regiones. Por un lado, se han establecido ciertas normas para tratar de impedir los fraudes y garantizar los productos alimenticios destinados a su transformación. Por otra parte, gracias a dispositivos técnicos y contractuales, se ha llevado a cabo una segmentación del mercado de consumo basada en el precio, el calendario, el aspecto y el origen de los productos.

Desde este punto de vista, la estandarización constituye un mecanismo crucial para entender el porqué de estas evoluciones como respuesta a la demanda masiva y al distanciamiento -espacial y temporal-entre los lugares de producción y consumo. Esta cuestión se impone progresivamente a partir de la segunda mitad del siglo XIX y parece garantizar el cumplimiento de los intereses sanitarios, económicos y sociales derivados de la expansión de los circuitos de suministro. Asimismo, se establecen normas y criterios comunes para las mercancías y para los métodos de conservación y empaquetado en el transporte. Estas normas, aunque controladas por instituciones científicas, organismos profesionales y administrativos, no pretenden únicamente clasificar los productos alimenticios para su transporte hacia los mercados o industrias. $\mathrm{Al}$ contrario, van más allá del proceso productivo con el objetivo de facilitar también las tareas de evaluación y control dentro de las explotaciones. Aunque cada cultivo especializado puede diferir del resto en términos de aplicación, las normas trazan los límites de un movimiento más general. Finalmente, acaban convirtiéndose en instrumentos capaces de controlar las tierras mediante las semillas (Bonneuil, 1999; Bonneuil y Thomas, 2009), así como los recursos naturales con la combinación de tratamientos fertilizantes y antiparasitarios (Fourche, 2004), o el trabajo familiar y salarial con la organización de las tareas y los ritmos de actividad (Berlan, 1986; Hubscher y Farcy, 1996; Morice y Michalon, 2008; Bernard de Raymond, 2011; Corrado, de Castro y Perrotta, 2016).

Los enfoques centrados en el metabolismo agrícola permiten ir más allá en el estudio de las evoluciones culturales en las explotaciones y de los equilibrios internos en las regiones (Le Noë, Billen y Garnier, 2017; Gingrich et al., 2018). Asimismo, muestran que la tendencia hacia el monocultivo no solo consiste en la utilización intensiva de recursos naturales o en la adopción de materiales y herramientas industriales. La demanda masiva de energías fósiles (mediante los insumos o la motomecanización) y el proceso de integración en los sectores agroindustriales provocan, sobre todo, la ruptura de las interacciones, complementariedades y sinergias -de carácter ecológico y económico-a largo plazo en el seno de los sistemas agrarios especializados, que tradicionalmente habían funcionado mediante los cultivos o las actividades pivot, tal y como se definen más arriba.

En relación con estas cuestiones, España ha sido un campo de estudio especialmente destacado durante los últimos años. Se han cuestionado las especializaciones desde el punto de vista de su huella ecológica y de su anclaje en las condiciones biorregionales (Garrabou et al., 2009; Duarte, Pinilla y Serrano, 2014; González de Molina et al., 2020). Por su parte, Juan Infante-Amate (2012) se centra en el caso del olivo español, en su transformación del paisaje y en sus diferentes usos a lo largo de tres siglos. Sus investigaciones muestran cómo este cultivo, símbolo de la "conquista campesina" del Mediterráneo, deja de ocupar tierras dispersas y pierde su carácter multifuncional (capaz de proporcionar al mismo tiempo madera, forrajes y materias primas para la protoindustria) para convertirse en una plantación intensiva con el único fin de producir aceite para el mercado nacional y para su exportación. Aunque la cantidad de biomasa extraída del olivo sigue siendo bastante estable, el autor muestra cómo los elementos que la componen varían enormemente durante el período analizado, en el que la producción de aceite se impone a las otras actividades competidoras. Esta especialización acaba por inscribirse en los mecanismos locales de gestión de recursos y se transforma en un monocultivo que depende cada vez más del exterior, para negociar su valor en los circuitos industriales y comerciales, pero también para restaurar sus ciclos frente a la degradación de los ecosistemas.

Así pues, el caso del olivo constituye un ejemplo de las trayectorias de especialización que retoman una producción determinada con el objetivo, por un lado, de impulsar prácticas más intensivas y, por otro, de 
asegurar un mejor control de los fenómenos estacionales y económicos. El verdadero impacto de este enfoque, no obstante, debe medirse a largo plazo, ya que "la agricultura de los monocultivos no saca partido de los principios por medio de los cuales opera la naturaleza; se trate de una agricultura como podría concebirla un ingeniero" (Carson, 2010, p. 10). Esta observación de Rachel Carson podría aplicarse al conjunto de actividades que durante tanto tiempo han motivado las especializaciones agrícolas. Igualmente, nos invita a contemplar el momento crucial de estos sistemas, así como las dinámicas de polarización experimentadas a lo largo del siglo XX.

\section{ConClusión}

Las especializaciones agrícolas pueden concebirse a la vez como un estado y como un proceso (Herment y Antoine, 2016). Los análisis centrados en el estado-descriptivos o en forma de indicio- dan cuenta de la situación en un instante preciso. Ante todo, permiten identificar las diferentes actividades abordadas y el lugar de cada una en la explotación. Su estructura permite considerar la integración de los procesos de producción $\mathrm{y}$, en algunos casos, comprender la inscripción de los cultivos especializados en los sistemas multifuncionales o de mixed farming. Para medir estos aspectos, Paul Brassley (2010) ha elaborado y probado un índice de especialización basado en los datos del Farm Management Survey, recogidos en Reino Unido desde 1936. Su solución da importancia al peso de la producción principal frente a las otras prácticas de la explotación. ${ }^{8}$ Esta perspectiva permite evaluar no solo el declive de la diversificación de cultivos, sino también el papel decisivo que puede adquirir un producto en el funcionamiento de las explotaciones. Sin embargo, es indispensable considerar este aspecto, sobre todo desde un punto de vista diacrónico, ya que las dinámicas contemporáneas dependen cada vez más de los agricultores, y no solo del rendimiento de una producción en particular. Así pues, este enfoque es realmente interesante. No obstante, a no ser que se repita con cierta regularidad, o no, y que tenga en cuenta la representatividad de las observaciones, no considera la evolución de los procesos de especialización en el tiempo, ni tampoco en relación con el medio.

$\mathrm{Al}$ contrario, la dimensión diacrónica de la especialización parte de su planteamiento en términos de proceso. Cabe diferenciar tres dimensiones diferentes. En primer lugar, las explotaciones especializadas se inscriben en los modos de producción que evolucionan con las innovaciones técnicas, la gestión de recursos y la integración de mercados. En segundo lugar, los procesos de especialización se sitúan en el tiempo y no pueden reducirse al inevitable declive de la variedad de cultivos dentro de la explotación; esta historia debería explicarse más en términos de regímenes sucesivos de especialización, caracterizados por diferentes formas y niveles de dependencia de los circuitos de suministro y de comercialización, de la acción institucional y de los sistemas agroindustriales. En tercer lugar, cada especialización se sitúa en un espacio construido por la misma y para la misma. Así pues, es necesario rastrear los paisajes remodelados por las transformaciones observables en las prácticas agrícolas, los flujos biofísicos y las infraestructuras de servicios.

Durante mucho tiempo, los principales focos de interés de la historiografía de los sistemas agrarios europeos han sido el tamaño de las explotaciones, la productividad de los cultivos y la rentabilidad de las inversiones. Asimismo, estas perspectivas analíticas se reflejan en la manera en que concebimos la emergencia y el desarrollo de los cultivos especializados. Sin embargo, durante las últimas décadas, con la aparición de nuevas preocupaciones medioambientales, cuestiones como la gestión, la circulación y la apropiación de recursos, tanto a nivel local como a gran escala, han alcanzado una importancia nunca antes vista (Moreno y Raggio, 1999; Cevasco y Tigrino, 2008). Si seguimos los pasos de la agroecología (Rosset y Altieri, 2017), el cambio de perspectiva que proponen estos enfoques contribuye a reforzar la hipótesis -mencionada en repetidas ocasiones- de que el territorio constituye el mejor marco de observación y seguimiento de los procesos de especialización agrícola. Así pues, más allá del funcionamiento de cada explotación, resulta cada vez más necesario recordar los orígenes, las evoluciones y las revoluciones que han hecho que ciertos espacios se organicen y se desarrollen al ritmo de una determinada producción. 


\section{ReFERENCIAS}

Allaire, G. (1996). Émergence d'un nouveau système productif en agriculture, Canadian Journal of Agricultural Economics, 44(4), 461-479.

Antoine, A. (1995). Les bovins de la Mayenne (1720-1820). Un modèle économique pour les campagnes de l'Ouest?. Histoire et sociétés rurales, 4, 105-136.

Antoine, A. (ed.) (2016). Agricultural Specialisation and Rural Patterns of Development. Turnhout: Brepols.

Aymard, M. (1983). Autoconsommation et marchés: Chayanov, Labrousse ou Le Roy Ladurie?. Annales. Économies, sociétés, civilisations, 38(6), 1392-1410.

Aymard, M. (1978). La transizione dal feudalesimo al capitalismo. En Storia d'Italia 1. Dal feudalesimo al capitalismo (pp. 1131-1192). Torino: Einaudi.

Bairoch, P. (1989). Les trois révolutions agricoles du monde développé: rendements et productivité de 1800 à 1985. Annales. Économies, sociétés, civilisations, 44(2), 317-353.

Barral, S. (2015). Capitalismes agraires. Économie politique de la grande plantation en Indonésie et en Malaisie. Paris: Presses de Sciences Po.

Béaur, G. (2009). Alternative Agriculture or Agricultural Specialization in Early Modern France?. En J. Broad (ed.), A Common Agricultural Heritage? Revising French and British Rural Divergence (pp. 121-137). Exeter: British Agricultural History Society.

Béaur, G. (2012). Histoire économique, histoire des campagnes: le renouveau d'un paradigme?. En J.-C. Daumas (ed.), L'histoire économique en mouvement: entre héritages et renouvellements (pp. 125-142). Villeneuve d'Ascq: Presses universitaires du Septentrion.

Béaur, G. (ed.) (2020). Alternative Agriculture in Europe (sixteenth-twentieth centuries). Turnhout: Brepols.

Becattini, G. (2000). "Distrettualità" fra industria e agricoltura. QA - La Questione Agraria, 2, 11-24.

Becattini, G. (2001). Distretti e ruralità: sfide al riduzionismo economico. Una replica. QA - La Questione Agraria, $1,119-127$.

Berlan, J.-P. (1986). Agriculture et migrations, Revue européenne de migrations internationales, 2(3), 9-32.

Bernard de Raymond, A. (2011). Une «Algérie californienne»? L'économie politique de la standardisation dans l'agriculture coloniale (1930-1962). Politix, 95, 23-46.

Bernard de Raymond, A. (2013). En toute saison. Le marché desfruits et légumes en France. Rennes: Presses universitaires de Rennes.

Bethemont, J. (1972). Le thème de l'eau dans la vallée du Rhône. Essai sur la genèse d'un espace hydraulique. SaintÉtienne: Imprimerie Le Feuillet Blanc.

Bevilacqua, P. (1989-1991). Storia dell'agricoltura italiana in età contemporanea. Venezia: Marsilio (Vol. 1-3).

Biagioli, G. (2019). La voie de la croissance agricole en Italie, XVI ${ }^{\mathrm{e}}$ siècle-1950. En L. Herment (ed.), Histoire rurale de l'Europe $X V I^{e}-X X^{e}$ siècle (pp. 69-93). Paris: Éditions de l'EHESS.

Bonneuil, C. (1999). «Pénétrer l'indigène»: arachides, paysans, agronomes et administrateurs coloniaux au Sénégal (1897-1950). Études rurales, 151-152, 199-223.

Bonneuil, C., Denis, G., y Mayaud, J.-L. (eds.) (2008). Sciences, chercheurs et agriculture. Pour une histoire de la recherche agronomique. Versailles: Éditions Quae, Paris: L'Harmattan.

Bonneuil, C., y Thomas, F. (2009). Gènes, pouvoirs et profits. Recherche publique et régimes de production des savoirs de Mendel aux OGM. Versailles: Éditions Quae, Lausanne: Fondation pour le progrès de l'Homme.

Bordas, J. (1954). Comment étudier le milieu cultural. Économie rurale, 19, 25-33.

Boserup, E. (1965). The Conditions of Agricultural Growth: The Economics of Agrarian Change under Population Pressure. London: Allen \& Unwin.

Brassley, P. (2010). Comments on the DEFRA Specialisation Index (Unpublished Working Paper), Centre for Rural Policy Research at the University of Exeter. 
Brunier, S. (2018). Le bonheur dans la modernité. Conseillers agricoles et agriculteurs, 1945-1985. Lyon: ENS Éditions. Cafagna, L. (1989). Dualismo e sviluppo nella storia d'Italia. Venezia: Marsilio.

Calatayud Giner, S. (1989). L'expansió citrícola valenciana. Producció i propietat de la terra a la Ribera del Xúquer (1850-1930). Recerques. Historia, Economia, Cultura, 22, 95-115.

Calatayud Giner, S. (2004). Canals d'innovació tecnològica a l'agricultura mediterrània occidental (1840-1930). Recerques. Història, Economia, Cultura, 49, 25-51.

Carson, R. (2010). Primavera silenciosa (J. Ros, trad.). Barcelona: Editorial Crítica. (Obra original publicada en 1962).

Cecchi, C. (1992). Per una definizione di distretto agricolo e distretto agroindustriale. QA - La Questione Agraria, 46, 81-107.

Celetti, D. (2007). La canapa nella Repubblica Veneta. Produzione nazionale e importazioni in età moderna. Venezia: Istituto Veneto di scienze, lettere ed arti.

Cevasco, R., y Tigrino, V. (2008). Lo spazio geografico: una discussione tra storia politico-sociale ed ecologia storica. Quaderni storici, 127, 207-242.

Chiapparino, F. (2015). Tra invenzione e tradizione. Note sulla storia delle tipicità dell'industria alimentare italiana. RiSES. Ricerche di storia economica e sociale, 1/2, 199-216.

Chiapparino, F., y Tedeschi, P. (eds.) (2017). Formaggi e vini. L'agroalimentare italiano tra industria e tipicità. Proposte e ricerche. Economia e società nella storia dell'Italia centrale, 79.

Cleaver, H. M. Jr (1972). The Contradictions of the Green Revolution. The American Economic Review, 62(1-2), 177-186.

Corrado, A., de Castro, C., y Perrotta, D. (eds.) (2016). Migration and Agriculture: Mobility and Change in the Mediterranean Area. New York: Routledge.

Cronon, W. (1991). Nature's Metropolis: Chicago and the Great West. New York-London: W. W. Norton \& Co.

Cruyningen, P. van (2019). Régulation des eaux, investissement urbain et développement de l'agriculture dans les provinces littorales des Pays-Bas, 1400-1900. En L. Herment (ed.), Histoire rurale de l'Europe XVI'-XXe siècle (pp. 47-68). Paris: Éditions de l'EHESS.

De Benedictis, M. (2000). Economia agraria e distrettualità. QA - La Questione Agraria, 2, 25-34.

Delfosse, C. (2007). La France fromagère, 1850-1990. Paris: La Boutique de l'histoire.

Delfosse, C. (ed.) (2011). La mode du terroir et les produits alimentaires. Paris: Les Indes savantes.

De Vries, J. (1974). The Dutch Rural Economy in the Golden Age, 1500-1700. Yale: Yale University Press.

Delhoume, J.-P. (2009). Les campagnes limousines au XVIII siècle. Une spécialisation bovine en pays de petite culture. Limoges: Presses universitaires de Limoges.

Dewerpe, A. (1985). L'Industrie aux champs. Essai sur la proto-industrialisation en Italie du Nord, 1800-1880. Roma: École française de Rome.

Dickinson, R. E. (1934). Markets and Market Areas of East Anglia. Economic Geography, 10(2), 172-182.

Drouard, A., y Williot, J.-P. (eds.) (2007). Histoire des innovations alimentaires XIX ${ }^{e}$ et XX $X^{e}$ siècles. Paris: L'Harmattan.

Dumont, R. (1952). Agriculture comparée. En Larousse Agricole (pp. 903-938). Paris: Larousse.

Duarte, R., Pinilla, V., y Serrano, A. (2014). The water footprint of the Spanish agricultural sector: 1860-2010. Ecological Economics, 108, 200-207.

Durbiano, C. (1997). Le Comtat et ses marges. Crises et mutations d'une région agricole méditerranéenne. Aix-enProvence: Publications de l'Université de Provence.

Durbiano, C. (ed.) (2000). Dynamiques spatiales des cultures spéciales. Méditerranée. Revue géographique des pays méditerranéens, 95.

Dyer, C. (2000). Review Article: Alternative Approaches to the History of Agriculture. Past \& Present, 168, $254-262$.

Failla, O., y Fumi, G. (eds.) (2006). Gli agronomi in Lombardia: dalle cattedre ambulanti ad oggi. Milano: Franco Angeli. 
Farcy, J.-C. (1986). Les paysans beaucerons au XIX'e siècle. Chartres: Sociétés Archéologiques d'Eure-et-Loir.

Federico, G. (1994). Il filo d'oro. L'industria mondiale della seta dalla Restaurazione alla grande crisi. Venezia: Marsilio.

Federico, G. (2005). Feeding the World: An Economic History of Agriculture, 1800-2000. Princeton: Princeton University Press.

Ferrer-Alòs, L. (2019). Agriculture, ressources naturelles et bien plus. Trois voies de la croissance agricole et d'industrialisation en Espagne, XVII ${ }^{\mathrm{e}}$-XIX ${ }^{\mathrm{e}}$ siècles. En L. Herment (ed.), Histoire rurale de l'Europe $X V I^{e}-X X^{e}$ siecle (pp. 111-130). Paris: Éditions de l'EHESS.

Fourche, R. (2014). Contribution à l'histoire de la protection phytosanitaire dans l'agriculture française (1880-1970) (Thèse de doctorat en Histoire), Université Lumière Lyon 2.

Garrabou, R., Tello, E., Cussó X., y Badia-Miró, M. (2009). Explaining agrarian specialization in an advanced organic economy. The province of Barcelona in the mid-nineteenth century. En V. Pinilla (ed.), Markets and Agricultural Change in Europe from the $13^{\text {th }}$ to the $20^{\text {th }}$ century (pp. 137-171). Turnhout: Brepols.

Garrabou, R., Tello, E., y Cussó, X. (2010). Ecological and socio-economic functioning in the middle of the nineteenth century. A Catalan case study (the Vallès county, 1850-1870). En E. Landsteiner y E. Langthaler (eds.), Agrosystems and Labour Relations in European Rural Societies (Middle Ages-Twentieth Century) (pp. 119-154). Turnhout: Brepols.

Garrido, S. (2010). Oranges or "Lemons"? Family Farming and Product Quality in the Spanish Orange Industry, 1870-1960. Agricultural History, 48(2), 224-243.

Garrier, G., y Hubscher, R. (1988). Entre faucilles et marteaux. Pluriactivités et stratégies paysannes. Paris: Presses universitaires de Lyon, Éditions de la Maison des Sciences de l'Homme.

Gingrich, S., Theurl, M. C., Erb, K., y Krausmann, F. (2018). Regional specialization and market integration: Agroecosystem energy transitions in Upper Austria, Regional Environmental Change, 18(4), 937-950.

Grantham, G. W. (1989). Agricultural Supply During the Industrial Revolution: French Evidence and European Implications. The Journal of Economic History, 49(1), 43-72.

Grantham, G. W. (1997). Espaces privilégiés: productivité agraire et zones d'approvisionnement des villes dans l'Europe préindustrielle. Annales. Histoire, sciences sociales, 52(3), 695-725.

Grantham, G. W. (1999). Contra Ricardo: On the macroeconomics of pre-industrial economies. European Review of Economic History, 3(2), 199-232.

González de Molina, M., Soto Fernández, D., Guzmán Casado, G., Infante-Amate, J., Aguilera Fernández, E., Vila Traver, J., y García Ruiz, R. (2020). The Social Metabolism of Spanish Agriculture, 1900-2008: The Mediterranean Way Towards Industrialization. Cham: Springer Open.

Hayami, Y., y Ruttan, V. W. (1971). Agricultural Development: An International Perspective. Baltimore-London: The John Hopkins University Press.

Head-König, A.-L. (2019). La croissance agricole en Suisse. Modalités, diversités régionales et décalages temporels, XVIII ${ }^{\mathrm{e}}$-milieu XX $\mathrm{X}^{\mathrm{e}}$ siècle. En L. Herment (ed.), Histoire rurale de l'Europe XVI ${ }^{e}-X X^{e}$ siècle (pp. 95-109). Paris: Éditions de l'EHESS.

Henriksen, I. (2009). The contribution of agriculture to economic growth in Denmark, 1870-1939. En P. Lains, y V. Pinilla (eds.), Agriculture and Economic Development in Europe Since 1870 (pp. 117-147). London: Routledge.

Henriksen, I., y O'Rourke, K. (2005). Incentives, technology and the shift to year-round dairying in late nineteenthcentury Denmark. Economic History Review, 58(3), 520-554.

Herment, L. (ed.) (2019). Histoire rurale de l'Europe XVI ${ }^{e}-X X^{e}$ siècle. Paris: Éditions de l'EHESS.

Herment, L., y Antoine, A. (2016). Specialisation in rural history: towards a definition. En A. Antoine (ed.), Agricultural Specialisation and Rural Patterns of Development (pp. 13-45). Thurnhout: Brepols.

Hoyle, R. W. (ed.) (2004). People, Landscape and Alternative Agriculture: Essays for Joan Thirsk. Exeter: British Agricultural History Society. 
Hubscher, R. (1979-1980). L'agriculture et la société rurale dans le Pas-de-Calais du milieu du XIX siècle à 1914. Arras: Mémoires de la Commission départementales de monuments historiques du Pas-de-Calais.

Hubscher, R., y Farcy, J.-C. (eds.) (1996). La moisson des autres. Les salariés agricoles aux XIX $X^{e}-X X^{e}$ siècles. Paris: Éditions Créaphis.

Iacoponi, L. (1990). Distretto industriale marshalliano e forme di organizzazione delle imprese in agricoltura. Rivista di Economia Agraria, 45(4), 712-734.

Iacoponi, L. (2000). Distrettualità agricola: una difficile (e breve) navigazione tra opposti paradigmi economici. $Q A$ - La Questione Agraria, 4, 111-118.

Infante-Amate, J. (2012). The Ecology and History of the Mediterranean Olive Grove: the Spanish Great Expansion, 1750-2000. Rural History, 23(2), $161-184$.

Infante-Amate, J. (2014). ¿Quién levantó los olivos? Historia de la especialización olivarera en elsur de España (ss. XVIII$X X)$. Madrid: Ministerio de Agricultura, Alimentación y Medio Ambiente.

Jones, R., y Dyer, C. (eds.) (2016). Farmers, Consumers, Innovators: The world of Joan Thirsk. Hatfield: University of Hertfordshire Press.

Karlinsky, N. (2005). California Dreaming: Ideology, Society, and Technology in the Citrus Industry of Palestine, 1890-1939. Albany: State University of New York Press.

Kautsky, K. (1899). Die Agrarfrage, eine Uebersicht über die Tendenzen der modernen Landwirthschaft und die Agrarpolitik der Sozialdemokratie. Stuttgart: J. H. W. Dietz Nachf.

Kopsidis, M., y Wolf, N. (2012). Agricultural Productivity Across Prussia During the Industrial Revolution: A Thünen Perspective. The Journal of Economic History, 72(3), 634-670.

Krugman, P. (1991). Increasing Returns and Economic Geography. Journal of Political Economy, 99(3), 483-499.

Lains, P., y Pinilla, V. (eds.) (2009). Agriculture and Economic Development in Europe Since 1870. London: Routledge.

Le Noë, J., Billen, G., y Garnier, J. (2017). How the structure of agro-food systems shapes nitrogen, phosphorus, and carbon fluxes: The generalized representation of agro-food system applied at the regional scale in France. Science of the Total Environment, 586, $42-55$.

Lorenzetti, L. (2010). Destini periferici. Modernizzazione, risorse e mercati in Ticino, Valtellina e Vallese, 1850-1930. Udine: Forum.

Lupo, S. (1990). Il giardino degli aranci. Il mondo degli agrumi nella storia del Mezzogiorno. Venezia: Marsilio.

MacFadyen, J. (2018). Flax Americana. A History of the Fibre and Oil that Covered a Continent. Montreal: McGillQueen's University Press.

Malthus, T. (1815). An Inquiry into the Nature and Progress of Rent, and the Principles by Which it is Regulated. London: John Murray.

Marshall, A. (1890). Principles of Economics. London: Macmillan.

Martini, M. (2000). L'expansion d'une culture commerciale. La production du chanvre dans la plaine de Bologne au XIX ${ }^{\mathrm{e}}$ siècle. Histoire \& Mesure, 15(3-4), 377-397.

Maat, H. (2001). Science Cultivating Practice: A History of Agricultural Science in the Netherlands and its Colonies, 1863-1986. Dordrecht: Kluwer Academic Publishers.

Mayaud, J.-L. (1999). La petite exploitation paysanne triomphante. France XIXe siècle. Paris: Belin.

Mazoyer, M., y Roudart, L. (1997). Histoire des agricultures du monde. Du néolithique à la crise contemporaine. Paris: Seuil.

Mesliand, C. (1989). Paysans du Vaucluse (1860-1939), Aix-en-Provence: Publications de l'Université de Provence, Aix-Marseille 1.

Moreno, D., y Raggio, O. (1999). Dalla storia del paesaggio agrario alla storia rurale. L'irrinunciabile eredità scientifica di Emilo Sereni. Quaderni storici, 100, 89-104.

Morice, A., y Michalon, B. (eds.) (2008). Travailleurs saisonniers dans l'agriculture européenne. Études rurales, 182. 
Moriceau, J.-M., y Postel-Vinay, G. (1992). Ferme, entreprise, famille. Grande exploitation et changements agricoles $X V I I^{e}-X I X^{e}$ siècles. Paris: Éditions de l'EHESS.

Morilla Critz, J. (ed.) (1995). California y el Mediterráneo. Estudios de la historia de dos agriculturas competidoras. Madrid: Ministerio de Agricultura, Alimentación y Medio Ambiente.

Morilla Critz, J., Olmstead, A. L., y Rhode, P. W. (1999). "Horn of Plenty": The Globalization of Mediterranean Horticulture and the Economic Development of Southern Europe, 1880-1930. The Journal of Economic History, 59(2), 316-352.

Morilla Critz, J., Olmstead, A. L., y Rhode, P. W. (2000). International competition and the development of the

dried fruit industry, 1880-1930. En S. Pamuk y J. Williamson (eds.), The Mediterranean Response to Globalization before 1950 (pp. 199-232). London-New York: Routledge.

Morineau, M. (1963). La productivité agricole du XIV ${ }^{\mathrm{e}}$ au XIX ${ }^{\mathrm{e}}$ siècle. Annales. Économies, sociétés, civilisations, 18(6), 1209-1212.

Moser, P., y Varley, T. (eds.) (2013). Integration through Subordination: the Politics of Agricultural Modernisation in Industrial Europe. Turnhout: Brepols.

Musotti, F. (2000). Il territorio: da sempre nell'analisi economico-agraria italiana. QA - La Questione Agraria, 4, 119-131.

Ouerfelli, M. (2008). Le sucre. Production, commercialisation et usages dans la Méditerranée médiévale. Leiden: Brill.

Pan-Montojo, J. (2005). Apostolado, profesión y tecnología. Una historia de los ingenieros agrónomos en España. Torrelodones: B\&H.

Pinilla, V. (ed.) (2009). Markets and Agricultural Change in Europe from the $13^{\text {th }}$ to the $20^{\text {th }}$ century. Turnhout: Brepols.

Pinilla, V., y Ayuda, M. I. (2009). Foreign markets, globalisation and agricultural change in Spain, 1850-1935. En V. Pinilla (ed.), Markets and Agricultural Change in Europe from the $13^{\text {th }}$ to the $20^{\text {th }}$ century (pp. 173-208). Turnhout: Brepols.

Ploeg, J. D. van der, Laurent, C., Blondeau, F., y Bonnafous, P. (2009). Farm diversity, classification schemes and multifunctionality. Journal of Environmental Management, 90, Supplement 2, S124-S131.

Poussou, J.-P. (1999). L'«agriculture alternative»? À propos d'un livre de Joan Thirsk. Histoire et sociétés rurales, 12, 131-147.

Poussou, J.-P. (2020). Another Look at Joan Thirsk's Concept of "Alternative Agriculture", and why it should be Discarded. En G. Béaur (ed.) (2020), Alternative Agriculture in Europe (sixteenth-twentieth centuries) (pp. 35-55). Turnhout: Brepols.

Quellier, F. (2003). Des fruits et des hommes. L'arboriculture fruitiere en Ile-de-France vers 1600-vers 1800. Rennes: Presses universitaires de Rennes.

Ricardo, D. (1817). On the Principles of Political Economy and Taxation. London: John Murray.

Rinaudo, Y. (1987). Un travail en plus: les paysans d'un métier à l'autre (vers 1830-vers 1950). Annales. Économies, sociétés, civilisations, 42(2), 283-302.

Ronsijn, W. (2019). Alternance d'effets de ciseaux dans l'espace rural de la Flandres intérieure, XVIII ${ }^{\mathrm{e}}$-XIX ${ }^{\mathrm{e}}$ siècle. En L. Herment (ed.), Histoire rurale de l'Europe XVI ${ }^{e}-X X^{e}$ siècle (pp. 203-230). Paris: Éditions de l'EHESS.

Rosset, P. M., y Altieri, M. A. (1997). Agroecology versus Input Substitution: A Fundamental Contradiction of Sustainable Agriculture. Society \& Natural Resources, 10(3), 283-295.

Rosset, P. M., y Altieri, M. A. (2017). Agroecology: Science and Politics. Winnipeg: Fernwood Publishing.

Segers, Y., Bieleman, J., y Buyst, E. (eds.) (2009). Exploring the Food Chain: Food Production and Food Processing in Western Europe, 1850-1990. Turnhout: Brepols.

Slicher van Bath, B. H. (1963). The Agrarian History of Western Europe, A. D. 500-1850. London: Edward Arnold.

Smits, J.-P. (2009). Technological change, institutional development and economic growth in Dutch agriculture, 1870-1939. En P. Lains y V. Pinilla (eds.), Agriculture and Economic Development in Europe Since 1870 (pp. 97-116). London: Routledge. 
Stanziani, A. (2005). Histoire de la qualité alimentaire, XIXe-XXe siècle. Paris: Seuil.

Thirsk, J. (1997). Alternative Agriculture: A History from the Black Death to the Present Day. Oxford: Oxford University Press.

Thirsk J. (1999). L'agriculture en Angleterre et en France de 1600 à 1800: contacts, coïncidences et comparaisons. Histoire Économie et Société, 18(1), 5-24.

Thünen, J. H. von [1826] (1875). Der isolirte Staat in Beziehung auf Landwirthschaft und Nationalökonomie. Berlin: Wiegandt, Hempel und Parey.

Tomich, D., dos Santos Gomes, F., y Gomes da Cunha, O. (eds.) (2011). Rethinking the Plantation: Histories, Anthropologies, and Archaeologies. Review (Fernand Braudel Center), 34(1/2).

Uekötter, F. (ed.) (2014). Comparing Apples, Oranges, and Cotton: Environmental Perspectives on the Global Plantation. Frankfurt-on-Main-New York: Campus Verlag.

Valceschini, E. (2016). Histoire de la régulation interprofessionnelle dans la filière des legumes transformés, des années 1960 aux années 1980. En A. Chatriot, F. Conord y É. Lynch (eds.), Orienter et réguler les marchés agricoles: entre pilotage national et politique agricole commune (pp. 91-109). Montreuil-sous-Bois: FranceAgriMer, Comité d'histoire des offices agricoles.

Vidal de La Blache, P. (1910). Régions françaises. La Revue de Paris, 17(6), 821-849.

Villani, P. (ed.) (1989). La pluriattività negli spazi rurali: ricerche a confronto. Bologna: Annali dell'Istituto Alcide Cervi, 11.

Williot, J.-P. (ed.) (2010). Approvisionnement ferroviaire et pratiques alimentaires des citadins. Revue d'histoire des chemins de fer, 41.

Wrigley, E. A. (2006). The transition to an advanced economy: half millennium of English agriculture. Economic History Review, 59(3), 435-480.

\section{Notas}

1 Las investigaciones de Edward Anthony Wrigley (2006) estudian la agricultura como proveedor de materias primas en la organic economy.

2 En Francia, Bernard H. Slicher van Bath (1963) tuvo un recibimiento muy discreto. Michel Morineau (1963) fue el único que prestó especial atención a su obra. Esta corriente de pensamiento no debe confundirse con el modelo de Hayami y Ruttan y los modelos de desarrollo endógeno más interesados por el papel de la producción en el marco de una actividad determinada (Hayamy y Ruttan, 1971). Para una comparación, véase Herment (2019).

3 Para una perspectiva bibliográfica, nos referimos a Herment y Antoine (2016) y a las obras arriba citadas de Jean-Pierre Poussou y Gérard Béaur. A estas referencias cabe añadir los artículos de Giuliana Biagioli (2019) sobre Italia, de Llorenç Ferrer-Alòs (2019) sobre España, de Jan-Pieter Smits (2009) y Piet van Cruyningen (2019) sobre los Países Bajos y de Anne-Lise Head-König (2019) sobre Suiza.

4 Aymard, 1983, p. 1409 (texto original en francés, aquí traducido al español).

5 En una reseña para la revista Historia Agraria (2019, 77, pp. 206-209), Piet van Cruyningen invita a Herment y Antoine (2016) a asimilar, en mayor o menor medida, la especialización como parte del proceso de comercialización. Desde nuestro punto de vista, esto le quita peso a la vertiente agraria de la especialización y a la necesidad de analizarla con precisión. Sin duda, la existencia de redes comerciales prueba la existencia de la especialización, pero no basta para caracterizarla, salvo que consideremos que las vacas pastan o caminan sobre el agua sin problema alguno, algo poco probable incluso en los Países Bajos en la Edad Moderna, como demuestran los trabajos de Jan de Vries (1974) y del propio Piet van Cruyningen (2019).

6 Si bien la ley de población es el eje de la argumentación ricardiana, su razonamiento no debe confundirse con el de Malthus, inspirado en la teoría de la renta y del valor del trabajo de Adam Smith.

7 Como indica Ester Boserup (1965), el crecimiento de la población no implica el cultivo de nuevas tierras, sino la intensificación de las prácticas agrícolas.

8 Por el contrario, existen otros indicadores que prefieren medir el nivel de concentración de margen bruto, es decir, la contribución de cada cultivo al resultado final. 